\title{
Biología floral y reproductiva de Escallonia pulverulenta (Ruiz et Pav.) Pers. (Escalloniaceae) y su relación con los visitantes florales
}

\section{Floral and reproductive biology of Escallonia pulverulenta (Ruiz et Pav.) Pers. (Escalloniaceae) and its relationship with floral visitors}

\author{
Antonio Rivera-Hutinel ${ }^{1 *} \&$ Fernando Acevedo-Orellana ${ }^{2}$ \\ ${ }^{1}$ Instituto de Entomología, Facultad de Ciencias Básicas, Universidad Metropolitana de Ciencias de la Educación, Av. José \\ Pedro Alessandri 774, Ñuñoa, Santiago, Chile. \\ ${ }^{2}$ Escuela de Agronomía, Facultad de Ciencias, Universidad Mayor, Camino La Pirámide 5750, Huechuraba, Santiago, Chile. \\ *antonio.rivera@umce.cl
}

\begin{abstract}
RESUMEN
Se determinó la biología floral y el sistema reproductivo de Escallonia pulverulenta, arbusto endémico de Chile central. Para ello se realizaron tratamientos de polinización manual, automática y natural a flores, evaluándose la producción de semillas por fruto. Además se determinó la longevidad floral, la producción de semillas según la distancia a la planta donante, los patrones de floración y su relación con los visitantes florales. La especie es autoincompatible y requiere vectores bióticos para reproducirse. Las plantas florecen sincrónicamente y logran el máximo despliegue floral a los 13 días de iniciar la floración. Cada flor queda disponible para ser polinizada durante 5 días y noches, y la fecundidad no incrementó con la distancia a la planta donante de polen. Las flores fueron visitadas por un ensamble diverso pero variable de polinizadores interanualmente. Debido a la longevidad de sus flores y su potencial reproductivo, esta planta podría utilizar una estrategia de floración masiva y atraer a un ensamble impredecible de polinizadores, los que luego de visitar las flores varias veces y durante varios días, logran adecuados niveles de polinización cruzada.
\end{abstract}

Palabras clave: Autoincompatibilidad, longevidad floral, distancia a la planta donante, polinizadores, matorral mediterráneo.

\begin{abstract}
This study examined the floral biology and reproductive system of Escallonia pulverulenta, an endemic shrub of central Chile. Manual, automatic and natural pollination treatments were performed, and seed set per fruit was evaluated. Also was evaluated floral longevity, seed production variation with the donor plant distance, the flowering patterns and its relationship with floral visitors. The species is self-incompatible and requires biotic vectors to reproduce. The flowers bloom synchronously and the floral display achieves their maximum thirteenth days after that flowering started. Each flower was available to be pollinated for five days and nights and fertility did not increase with distance from the pollen donor plant. The flowers were visited by a diverse assemblage of pollinators but variable between years. Because the longevity of their flowers and their reproductive potential, this plant could use a mass-flowering strategy and attract an unpredictable assembly of pollinators, which after visiting several times over several days the flowers could reach enough levels of cross pollination.
\end{abstract}

KEYwORDS: Self-incompatibility, floral longevity, distance to the donor plant, pollinators, Mediterranean scrub.

\section{INTRODUCCIÓN}

El sistema reproductivo de las plantas influye en la estructura genética de las poblaciones y en su potencial reproductivo (Barrett 2003). Diferentes estudios han encontrado relación entre la proximidad espacial y el nivel de consanguinidad de los individuos, producto de una dispersión a corta distancia de polen $y / o$ semillas, lo que resulta en poblaciones espacialmente estructuradas (Glaettli et al. 2006). Como resultado, el apareamiento entre vecinos puede generar una fertilidad reducida en comparación con apareamientos entre individuos más distantes, debido a un incremento de los niveles de homocigosidad (Byers 1998, Hardner et al. 1998, Glaettli et al. 2006). De este modo, la polinización cruzada entre individuos genéticamente diferentes (i.e. xenogamia) incrementa las variaciones genéticas entre individuos y su 
potencial de adaptación al ambiente, aunque su costo es la pérdida de potencial reproductivo si los polinizadores son escasos (Lloyd \& Schoen 1992, Eckert \& Barrett 1994, Charlesworth 2002). Por otro lado, la autopolinización por polen de la misma flor (i.e. autogamia) o por polen de otras flores de un mismo individuo o clon (i.e. geitonogamia) representan para la planta un mecanismo de aseguramiento reproductivo frente a una disminución de agentes polinizadores, aunque implica un incremento del riesgo de depresión por endogamia por la expresión de genes deletéreos (Glover 2014) y un mayor descuento de polen, lo que se traduce en una disminución del polen disponible para la polinización cruzada (Barrett 2002).

En plantas hermafroditas, los mecanismos que evitan o disminuyen la autopolinización corresponden a la separación espacial (i.e. hercogamia) y/o temporal (i.e. dicogamia) entre las anteras y el estigma, además de la autoincompatibilidad genética y heteromórfica (Bertin 1993, Barrett 2002, McClure \& Franklin-Tong 2006, Takebayashi et al. 2006). La autoincompatibilidad implica un reconocimiento del acervo genético del grano de polen y la interrupción de la fecundación del óvulo cuando el polen proviene de la misma planta o de un individuo genéticamente similar (Barrett 2002). Este mecanismo obliga a que la fecundación se produzca solo si el transporte del polen por vectores se realiza a distancias donde pueda ocurrir variación genética efectiva entre individuos no emparentados, reduciendo la endogamia y asegurando la adecuación biológica de la planta (Schmitt \& Gamble 1990). En general, las plantas leñosas y longevas tienden a ser autoincompatibles mientras que las herbáceas anuales tienden a ser autocompatibles (Arroyo \& Squeo 1990, Barrett 2003).

Atributos de la biología floral, como el período de floración, el tiempo de receptividad del estigma, el despliegue floral, la maduración de las anteras y la longevidad floral, influyen en la transferencia de polen por los polinizadores, en las tasas de geitonogamia y en el tipo de polinizador asociado (Primack 1980, 1985, Zimmerman 1988, Lloyd \& Schoen 1992, Harder \& Barrett 1995, Barrett 2003). Por ejemplo, especies protándricas son frecuentemente polinizadas por abejas o moscas, mientras que las protoginias son polinizadas por escarabajos o por el viento (Sargent \& Otto 2004). Esto sugiere que la conducta de forrajeo del polinizador puede tener importantes consecuencias en la efectividad de las estrategias reproductivas para minimizar la autopolinización. Por otro lado, la floración es un factor decisivo en la reproducción de las plantas. Responde a fuerzas selectivas como la regulación de flujo polen, conducta de forrajeo de los polinizadores, hábitat y recursos disponibles (Primack 1985). La floración puede presentarse en masa, con individuos produciendo un gran número de flores en un período de tiempo corto o puede ser presentada de forma constante, con individuos produciendo pocas flores diariamente por períodos prolongados (Augspurger 1983).
Mientras la floración en masa generalmente se asocia a un gran número de polinizadores no especializados $\mathrm{y}$ oportunistas, un florecimiento constante o extendido puede alimentar a una población de un polinizador específico y especialista durante un período de tiempo prolongado (Yumoto 1987), lo que además podría incidir en diferentes niveles de geitonogamia (Augspurger 1983, De Jong et al. 1992). Por lo tanto, es necesario conocer la biología floral, la fenología de la floración, el sistema reproductivo y el agente polinizador que posee una especie para comprender de mejor forma aspectos de su biología reproductiva (Primack 1980, 1985).

El género Escallonia, el más diverso de la familia Escalloniaceae (48 especies, The Plant List 2013) y de distribución neotropical (Sleumer 1968), está representado en Chile por trece especies leñosas: doce distribuidas en el territorio continental y una en el Archipiélago de Juan Fernández (Zapata 2013), de las cuales siete especies son endémicas de Chile (MMA 2016). Morfológicamente, las especies de Escallonia van desde subarbustos $(\sim 1 \mathrm{~m})$, $\operatorname{arbustos}(<5 \mathrm{~m})$ a pequeños árboles $(\sim 6 \mathrm{~m})$. Las flores pueden ser solitarias o encontrarse en cimas o racimos de número variable, con formas discoidales, acampanadas o tubulares y de color verde, blanco, rosado o rojo, lo que sugiere la presencia de diferentes vectores de polen, pero muy poco se sabe sobre este aspecto en la mayoría de las especies de Escallonia (Zapata 2013). A la fecha, el único estudio que da cuenta de la biología reproductiva y los visitantes florales de una especie de Escallonia fue realizado por Valdivia \& Niemeyer (2006), en E. myrtoidea Bertero ex DC. En este estudio se determinó que presenta autoincompatibilidad y que sus potenciales polinizadores serían abejas.

El presente trabajo tiene por objetivo determinar el sistema reproductivo, los patrones de floración, la longevidad floral y su relación con los visitantes florales de Escallonia pulverulenta (Ruiz et Pav.) Pers. a fin de ampliar el conocimiento de la biología reproductiva y la polinización de las especies del género. Se espera que E. pulverulenta sea autoincompatible al igual que la especie congénere E. myrtoidea. También se espera que sean principalmente visitadas por abejas, ya que es considerada una planta de importancia apícola por la miel monofloral que se produce en Chile central (Montenegro et al. 2010).

\section{MATERIALES Y MÉTODOS}

\section{ESPECIE Y SITIO DE ESTUDIO}

Escallonia pulverulenta es un árbol o arbusto endémico de Chile central, que habita entre las regiones de Coquimbo y de La Araucanía $\left(30^{\circ}\right.$ a $\left.39^{\circ} \mathrm{S}\right)$ en bordes de quebradas y laderas secas (Hoffmann 2012). Las flores son hermafroditas y se encuentran agrupadas en inflorescencias racemosas alargadas y compactas (Fig. 1a). Florece en el verano austral 
entre noviembre y febrero, dependiendo de su ubicación geográfica (Kausel 1953). El fruto es una cápsula leñosa dehiscente (Fig. 1b) que se abre dispersando sus semillas en marzo, cada una de $0,8 \mathrm{~mm}$ de largo (Fig. 1c). Detalles sobre la estructura del nectario se pueden encontrar en DíazForestier et al. (2016).

El estudio se realizó en la Reserva Nacional Rio Clarillo, ubicada en la comuna de Pirque, Región Metropolitana (334' $\left.\mathrm{S}-70^{\circ} 27^{\prime} \mathrm{O}\right)$, desde noviembre de 2013 a enero de 2016. La Reserva en su zona más baja presenta una vegetación conformada por bosque esclerófilo y matorral xérico. El clima de la zona es mediterráneo semiárido con tendencias continentales y marcada oscilación estacional (Teillier et al. 2005). En la Reserva, E. pulverulenta es más abundante a altitudes menores a 1.200 m s.n.m. y en laderas de exposición norte, principalmente en los márgenes del bosque esclerófilo.

\section{MORFOLOGÍA FLORAL}

Para determinar la morfología floral se tomaron fotos de un total de 30 flores, provenientes de 3 diferentes alturas de cada planta en 10 plantas diferentes. Para cada medición, en laboratorio se obtuvo una foto de cada flor con regla graduada y se analizó con el programa ImageJ versión 1.6 (Schneider et al. 2012). Se midió (en mm): 1) el largo de los pétalos, 2) el ancho del nectario, 3) el largo del ovario, 4) la distancia entre la base del nectario y la superficie del estigma, denominada distancia operacional (DO), 5) la distancia promedio entre estambres vecinos (DEE), 6) la distancia promedio entre los estambres y el estigma (DPE) y 7) el ángulo de apertura (Aaper, en grados), correspondiente al ángulo que se forma entre el estigma, la base del nectario y el pétalo (ver Fig. 1d).

\section{LONGEVIDAD FLORAL}

Para estimar la longevidad floral se definieron fases florales, las que se identificaron en base a los cambios físicos que experimentaron las flores desde el estado de botón hasta la senescencia. Para esto se fotografió regularmente 12 flores numeradas de 12 plantas diferentes y se registraron los cambios en posición, tamaño, coloración y textura de las estructuras florales, como la fecha y hora, longevidad, posición del estigma y de los estambres. Además, se evaluaron los cambios temporales en la receptividad del estigma en 4 plantas, utilizando 6 flores emasculadas por planta y se realizó polinización cruzada manual utilizando polen de plantas vecinas en un radio de $5 \mathrm{~m}$. Se evaluó la receptividad del estigma al día $0,1,3,6,7$ y 8 desde la antesis floral (etapa F1). Las flores tratadas fueron embolsadas y se retiraron los frutos a principio de marzo, para luego contar el número de semillas por fruto en cada tratamiento bajo lupa con el programa ImageJ. Una disminución en la producción de semillas con la longevidad floral se consideró como pérdida de receptividad estigmática.
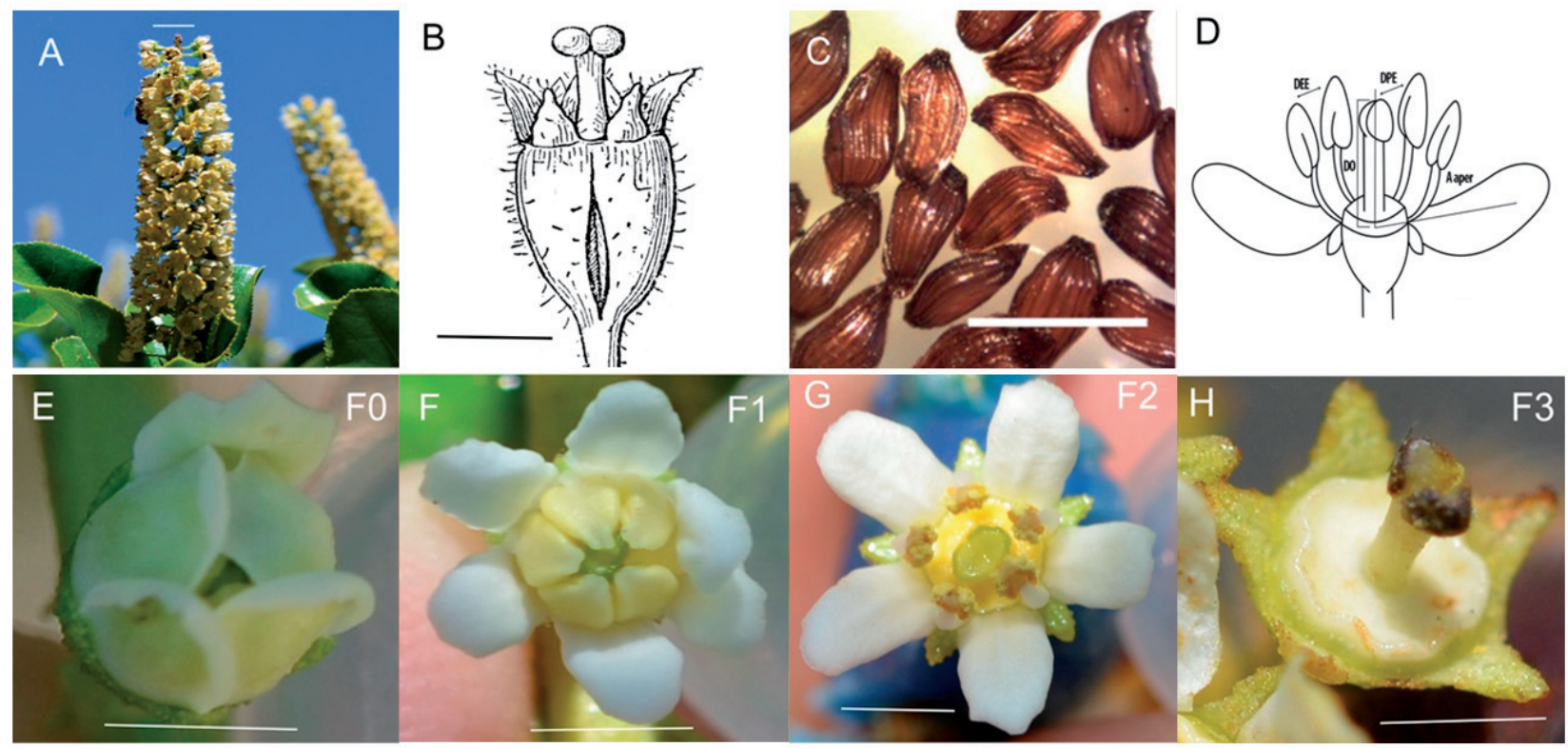

Figura 1. Etapas del desarrollo floral (fases) de E. pulverulenta. A) Inflorescencia (línea = $1 \mathrm{~cm}$ ), B) fruto dehiscente (línea = $2 \mathrm{~mm}$ ), C) Semillas (línea $=1 \mathrm{~mm}$ ), D) Esquema floral con medidas morfológicas (DEE=distancia entre estambres, DPE=distancia antera-estigma, $\mathrm{DO}=$ distancia operativa, Aaper=Angulo de apertura), E) botón floral en etapa pre-antesis (F0), línea = $2 \mathrm{~mm}, \mathrm{~F})$ flor en antesis con antera indehiscente (F1), línea $=2 \mathrm{~mm}, \mathrm{G})$ flor homógama $(\mathrm{F} 2)$, línea $=2 \mathrm{~mm}, \mathrm{H}$ ) Flor senescente (F3), línea $=2 \mathrm{~mm}$. / Floral development stages (phases) of E. pulverulenta. A) Inflorescence (line $=1 \mathrm{~cm}$ ), B) dehiscent fruit (line $=2 \mathrm{~mm}$ ), C) Seeds (line $=1 \mathrm{~mm}), \mathrm{D}) \mathrm{Scheme}$ flower with morphological measurements $(\mathrm{DEE}=$ distance between stamens, $\mathrm{DPE}=$ distance anther-stigma, $\mathrm{DO}=$ operating distance, Aaper $=$ opening angle), E) floral button at pre-anthesis stage (F0), line $=2 \mathrm{~mm}, \mathrm{~F}$ ) flower in anthesis with indehiscent anther $(\mathrm{F} 1)$, line $=2 \mathrm{~mm}, \mathrm{G})$ flower homogamous (F2), line $=2 \mathrm{~mm}, \mathrm{H})$ senescent flower fase (F3), line $=2 \mathrm{~mm}$. 
Fenología

Para estimar la fenología de E. pulverulenta se observó durante una temporada la floración de 15 individuos (diciembre 2013-enero 2014) de forma diaria. En cada individuo se contó el número de inflorescencias totales y el porcentaje de inflorescencias con flores en antesis. Para el análisis de la sincronía de la floración se utilizó el método de Primack (1980), donde el grado de sincronía entre dos individuos $\mathrm{i}$ y $\mathrm{j}$ viene dado por $\mathrm{c}_{\mathrm{ij}}=\mathrm{a} / \mathrm{b}$, siendo a el tiempo común de floración, mientras que $\mathrm{b}$ es el tiempo total de floración considerando ambas plantas.

El nivel de sincronía medio de los individuos $(Z)$ se calculó como $\mathrm{Z}=\Sigma \mathrm{c}_{\mathrm{ij}} / \mathrm{N}$, donde $\mathrm{N}$ es él número de comparaciones realizadas $[\mathrm{N}=\mathrm{n}(\mathrm{n}-1) / 2]$, siendo $\mathrm{n}$ el número de individuos. El nivel de sincronía para cualquier par de plantas adquiere un valor comprendido entre 0 (i.e. asincrónico) y 1 (i.e. sincrónico). Para evaluar si E. pulverulenta presentó una floración sincrónica, se comparó con test de Chi cuadrado la distribución de frecuencia del nivel de sincronía observado con el esperado bajo un patrón de floración aleatorio, lo que se hizo permutando los períodos de floración de cada planta 100 veces.

\section{SisteMA REPRODUCTIVO}

Con el fin de conocer el sistema reproductivo de $E$. pulverulenta, se realizaron experimentos de polinización para evaluar la posterior producción de frutos y semillas (Kearns \& Inouye 1993). Cada tratamiento fue evaluado en 2 a 3 flores de diferentes inflorescencias en 11 plantas diferentes, resultando un total de 32 a 33 flores evaluadas por tratamiento, los que se detallan a continuación: 1) agamospermia (AGA): Corresponde a flores emasculadas al comienzo de la antesis floral y aisladas de los polinizadores hasta la formación del fruto, 2) autopolinización espontánea (AUTO): flores sin emascular que se mantuvieron embolsadas desde el estado de botón aisladas de los polinizadores, con el fin de evaluar la capacidad de autopolinizarse en su ausencia (i.e. autogamia), 3) autopolinización manual (APM): flores emasculadas que se polinizaron manualmente con polen de la misma planta y luego se mantuvieron embolsadas (i.e. geitonogamia), 4) polinización cruzada manual (PCM): Flores emasculadas y polinizadas manualmente con polen proveniente de plantas vecinas dentro de $5 \mathrm{~m}$ de distancia desde la planta receptora, las que posterior al tratamiento fueron embolsadas nuevamente (i.e. xenogamia) y 5) polinización natural (PN): flores marcadas que se dejaron expuestas a los polinizadores todo el tiempo. En este último caso se marcaron 7 flores en cada una de las 11 plantas utilizadas en los otros tratamientos.

Los frutos fueron recuperados en marzo y se contó el número de semillas bajo lupa con el programa ImageJ. Se calculó el índice de autoincompatibilidad (IAI), que va de 0 (i.e. autoincompatibilidad) a 1 (autocompatibilidad), a través de la razón entre el número promedio de semillas por flor producidas por autopolinización manual dividido por polinización cruzada manual (Ruiz-Zapata \& Arroyo 1978). Se consideró a la planta como autoincompatible si IAI < 0,2 (Arroyo \& Squeo 1990). También se evaluó la eficiencia reproductiva (ER) como la razón entre las semillas producidas bajo polinización natural respecto a las producidas por polinización cruzada manual. Valores cercanos a 1,0 indican que los polinizadores están presentes de forma adecuada, mientras que valores $<1,0$ indican limitación de polen mediada por polinizadores (Bierzychudek 1981, Young \& Young 1992). Por último, se calculó el índice de autofertilidad como la razón entre el número de semillas producidas por polinización automática y polinización cruzada manual (Lloyd \& Schoen 1992). En los 3 casos se calculó un valor promedio de cada índice obtenido para cada planta $(n=11)$ y se evaluó si difería significativamente de 0 ó 1 por test de Wald (Fox 2002).

Distancia a la Planta donAnte

Para evaluar el efecto de la distancia desde donde proviene el polen en el éxito reproductivo de E. pulverulenta, se comparó la producción de semillas en flores polinizadas manualmente con polen de la misma planta $(=0 \mathrm{~m})$ y con polen proveniente de plantas a distancias de $5 \mathrm{~m}, 30 \mathrm{~m}$ y $1.000 \mathrm{~m}$ de distancia. Los tratamientos con polen proveniente de plantas distanciadas a $0 \mathrm{~m}$ y $5 \mathrm{~m}$ correspondieron a los tratamientos de autopolinización manual $(0 \mathrm{~m})$ y polinización manual cruzada $(5 \mathrm{~m})$, de los ensayos de biología reproductiva del punto anterior. Los tratamientos con polen proveniente de plantas distanciadas a $30 \mathrm{~m}$ y $1.000 \mathrm{~m}$ de la planta receptora del polen se realizaron en 2 a 3 flores de las 11 plantas utilizadas en los ensayos de biología reproductiva, que se agregaron a los ensayos de APM $(=0 \mathrm{~m})$ y PCM $(=5 \mathrm{~m})$. Una vez realizada la polinización manual se embolso la flor.

Los resultados se evaluaron mediante el recuento de frutos y semillas producidos al inicio de la temporada de fructificación (marzo). Estos frutos se mantuvieron embolsados hasta la etapa predispersiva, y luego fueron llevados al laboratorio.

\section{FRECUENCIA DE VISITANTES FLORALES}

Para estimar posibles variaciones interanuales en la identidad $\mathrm{y}$ frecuencia de visitas de los visitantes florales se realizaron un total de 2379 (198,25 h), 1000 (83,3 h) y 729 (60,75 h) observaciones por año a 40 plantas durante 3 temporadas (años 2013, 2014 y 2015). En cada observación se registraron todos los visitantes florales que hicieron contacto con las estructuras reproductivas de las flores de E. pulverulenta durante $5 \mathrm{~min}$, los que fueron capturados para su posterior identificación en caso de ser necesario. Las observaciones fueron realizadas en días soleados entre las 8:00 a.m. y las 20:00 p.m., abarcando un área de $\sim 5$ ha para detectar diferencias en los visitantes que reciben (Herrera 2005). Para observar polinizadores nocturnos se registró sólo en la última 
temporada (año 2015) durante tres noches entre las 22:00 p.m. y las 2:00 a.m., realizando un total de 49 observaciones (4,08 h de observación). La frecuencia de visitas de las especies nocturnas se calculó proporcional a la cantidad de observaciones realizadas de día respecto de noche.

\section{ANÁLISIS ESTADísticos}

Debido a que los diferentes tratamientos de receptividad del estigma, sistema reproductivo y distancia a la fuente de polen fueron realizados en diferentes flores de varios individuos de E. pulverulenta, los datos fueron analizados con ANDEVA de medidas repetidas considerando los individuos como la unidad de análisis, con el fin de asegurar la independencia de los datos y evitar pseudorreplicación sacrificada (Hurlbert 1984). Cuando se detectaron diferencias significativas entre tratamientos se utilizó test de Tukey como prueba de contrastes múltiples. Se utilizó como variable respuesta la fracción de flores que pasan a frutos y el número de semillas por fruto, los que fueron transformados a logaritmo neperiano para cumplir con los supuestos de normalidad y homocedasticidad de ANDEVA. Los análisis fueron realizados en el software PAST v3.14 (Hammer et al. 2001).

Para el caso de la floración, los cálculos de sincronía floral entre plantas $\left(\mathrm{c}_{\mathrm{ij}}\right)$ y nivel de sincronía medio $(\mathrm{Z})$ se realizaron en el software R v3.3.0 (R Development Core Team 2009). Para evaluar si la sincronía floral observada difiere del azar, se permutaron los días en floración manteniendo fijo el número de días que cada planta permanece en floración. Se utilizó test de Chi cuadrado para evaluar si la frecuencia de valores de sincronía observados difiere de lo esperado por el azar.

\section{RESULTADOS}

\section{Morfología FlORAL}

Las flores de E. pulverulenta son hermafroditas, actinomorfas, pentámeras, de 5 a $7 \mathrm{~mm}$ de largo. El cáliz es gamosépalo, persistente en el fruto formado por cinco lacinias pestañosas de color verde oscuro. La corola es dialipétala con cinco pétalos de color blanco, de largo 3,67 $\pm 0,05 \mathrm{~mm}$ (media $\pm 1 \mathrm{EE}, \mathrm{n}=28$ flores). Los pétalos

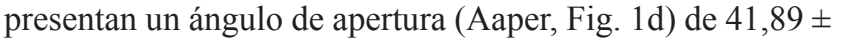
$0,25^{\circ}$ (media $\pm 1 \mathrm{EE}, \mathrm{n}=28$ flores) en las flores ubicadas al centro de la inflorescencia, pero pueden llegar a $90^{\circ}$ en las flores basales. El ancho de la base de la flor, donde se ubica el disco nectarífero de color amarillo, fue de 2,22 $\pm 0,04 \mathrm{~mm}$ (media \pm 1 EE, $\mathrm{n}=28$ flores). El androceo está conformado por 5 estambres libres, separados entre sí (DEE, Fig. 1d) por $1,61 \pm 0,01 \mathrm{~mm}$ (media $\pm 1 \mathrm{EE}, \mathrm{n}=28$ flores). El filamento es blanco y posee una longitud de $2 \mathrm{~mm}$. Las anteras son oblongas y poseen 2 tecas amarillas que presentan dehiscencia longitudinal introrsa al madurar. La unión entre el filamento y la antera es basifija. Las anteras se disponen a la misma altura que el estigma, con una distancia anteraestigma (DPE, ver Fig. 1d) de 1,54 $\pm 0,02 \mathrm{~mm}$.

El ovario es ínfero, sincárpico, con estigma bilobulado de color blanquecino-verdoso, de $1 \mathrm{~mm}$ de ancho (Fig. 1g). El estigma se encuentra distanciado de la base de la flor (DO, Fig. 1d) por 2,28 $\pm 0,02 \mathrm{~mm}$ (media $\pm 1 \mathrm{EE}, \mathrm{n}=28$ flores). El ovario es bilocular globoso de largo 2,02 $\pm 0,04 \mathrm{~mm}$ (media $\pm 1 \mathrm{EE}, \mathrm{n}=28$ flores), con varios óvulos. El número máximo de óvulos contabilizados en 10 flores fue de 198.

Las flores se encuentran agrupadas en inflorescencias racemosas o paniculadas, como espiga terminal densa, de largo variable, entre 4,2 y $22 \mathrm{~cm}$ de largo $(12,05 \pm 1,63 \mathrm{~cm}$, media $\pm 1 \mathrm{EE}, \mathrm{n}=10$ inflorescencias $)$. La cantidad de flores en el raquis de la inflorescencia es $10,77 \pm 0,72$ flores/cm de raquis (test de regresión lineal, pendiente $\left.\pm 1 \mathrm{EE}, \mathrm{r}^{2}=0,75 ; \mathrm{t}=4,92 ; \mathrm{p}=0,001\right)$.

\section{LONGEVIDAD FLORAL}

La fase previa a la antesis floral se denominó F0. Se reconocieron otras 3 fases fenológicas, identificables por la orientación y color de los pétalos y estambres, además de la coloración del estigma. Estas fases se detallan a continuación: F0) pre-antesis: el gineceo y el androceo se encuentran totalmente cubiertos por la corola. En esta fase los pétalos, ya totalmente distinguibles en el botón floral, convergen hacia el centro de la flor sobreponiéndose entre sí y las estructuras reproductivas no son visibles (Fig. 1e). Esta fase dura 2,20 $\pm 0,31$ días (media $\pm 1 \mathrm{EE}, \mathrm{n}=4$ flores), F1) antesis con anteras indehiscentes: Esta fase tiene una duración de 1,54 \pm 0,19 días (media $\pm 1 \mathrm{EE}, \mathrm{n}=12$ flores). La flor inicia su apertura, distinguible por la apertura de la corola. Las anteras, aún indehiscentes, se encuentran cubriendo el estigma orientadas hacia el centro de la flor (Fig. 1f). Inicialmente los filamentos están erectos, en un ángulo de $90^{\circ}$ respecto a la base de la flor y lentamente adoptan un ángulo agudo, dejando el estigma visible. F2) flor homógama: Esta fase tiene una duración de 5,73 \pm 0,33 días (media $\pm 1 \mathrm{EE}, \mathrm{n}=10$ flores) y se caracteriza porque la flor es funcionalmente masculina y femenina al mismo tiempo. El estigma continúa receptivo desde la etapa anterior y las anteras, ya distanciadas del estigma pasan a ser dehiscentes de color amarillo. Esta es la fase funcional para la polinización porque puede donar y recibir polen, $\mathrm{y}$ F3) Flor senescente: Esta fase comienza a los 7,27 $\pm 0,26$ días desde F1 y se caracteriza por el marchitamiento de los pétalos, los que se vuelven levemente amarillos. El estigma comienza a tornarse de color negro y deja de ser receptivo. Las anteras pierden el polen y se tornan café. Con el paso de los días terminan cayendo los pétalos y estambres, mientras el estilo persiste en el fruto en formación.

En relación a las pruebas de receptividad del estigma, se encontró que solo al octavo día disminuye la receptividad estigmática (AMR: $\mathrm{F}(5,15)=3,85 ; \mathrm{p}=0,019)$, obteniendo 
un número de semillas por fruto baja (media $\pm 1 \mathrm{EE}=3,00 \pm$ 2,12, Tabla 1). Sin embargo, el número de semillas por fruto no difirió significativamente entre el día inicial y el séptimo día (test de Tukey, p > 0,2 en todos los casos). En base al seguimiento de flores marcadas, al octavo día desde el inicio de la antesis (F1) comienza la fase de senescencia (F3), por lo que es esperable una disminución de la receptividad estigmática.

El estigma comienza a ser receptivo en la fase F1 (día 0 y 1), aun cuando las anteras inmaduras cubren al estigma y al nectario, quedando inaccesible para los visitantes florales, por lo que en esta etapa no se produciría polinización cruzada de forma natural. Por otro lado, la máxima receptividad del estigma se encontró al tercer día del ensayo (media \pm 1 $\mathrm{EE}=67,63 \pm 15,93$ semillas por fruto), al comienzo de la fase F2, cuando la flor homógama se encuentra totalmente expuesta a los polinizadores. Sin embargo, esta fecundidad no fue significativamente diferente al compararla con los tratamientos a los 0, 1, 6 y 7 días (Tabla 1).

\section{Fenología}

Dentro de una inflorescencia, las flores comienzan la antesis desde la base hacia el ápice, por lo que en el transcurso de la floración se observan flores en distintas fases florales dentro de cada inflorescencia y en cada individuo. La duración de la floración por planta fue de 29,33 $\pm 1,61$ días (media \pm $1 \mathrm{EE}, \mathrm{n}=15$ ). El máximo de floración se alcanzó 13,26 \pm 0,84 días después que cada planta empezó a florecer. El nivel de sincronía floral entre plantas fue de 0,69 $\pm 0,02$ (media $\pm 1 \mathrm{EE}, \mathrm{n}=15$ ), con un rango entre 0,05 y 0,97 . Al comparar la distribución de sincronía floral entre plantas $\left(\mathrm{c}_{\mathrm{ij}}\right)$ con la obtenida si las plantas florecieran al azar (Fig. 2), se encontraron diferencias significativas entre la frecuencia esperada y observada (Chi cuadrado $=993,41 ; \mathrm{gl}=9 ; \mathrm{p}=$ $0,001)$. Estas diferencias indican que los individuos de $E$. pulverulenta florecen más sincrónicamente entre sí que lo esperado por el azar (Fig. 2).

\section{SisTEMA REPRODUCTIVO}

Los tratamientos de polinización presentaron diferencias en la fracción de frutos formados (AMR: $\mathrm{F}(4,40)=37,48 ; \mathrm{p}<$ $0,001)$ y formación de semillas por fruto $(A M R: F(4,40)=$ $58,10 ; \mathrm{p}<0,001)$. Las flores de la prueba de agamospermia fructificaron en un bajo porcentaje $(4,54 \%)$ y con un bajo número de semillas $(0,13 \pm 0,13$ semillas/fruto), por lo que la especie puede ser considerada como no apomíctica (Tabla 2). La fructificación y número de semillas por fruto producidas por autopolinización automática fue 8,16 veces y 53,45 veces menores que las obtenidas por polinización cruzada manual, respectivamente (test de Tukey: $\mathrm{Q}_{\text {frutos }}=$ 10,$12 ; \mathrm{P}_{\text {frutos }}<0,001 ; \mathrm{Q}_{\text {semillas }}=13,00 ; \mathrm{P}_{\text {semillas }}<0,001$, Tabla 2), mientras que la fructificación y número de semillas por fruto producidas por autopolinización manual fue 1,6 veces y 14,2 veces menores que las obtenidas por polinización cruzada manual, respectivamente (test de Tukey: $\mathrm{Q}_{\text {frutos }}=$ 4,$21 ; \mathrm{P}_{\text {frutos }}=0,037 ; \mathrm{Q}_{\text {semillas }}=11,16 ; \mathrm{P}_{\text {semillas }}<0,001$, Tabla 2). Por otro lado, la fructificación y número de semillas por fruto producidas por polinización cruzada manual fue 1,33 veces y 1,28 veces menor que la polinización natural, sin diferencias significativas entre ambas (test de Tukey: $Q_{\text {frutos }}$ $=3,07 ; \mathrm{P}_{\text {frutos }}=0,21 ; \mathrm{Q}_{\text {semillas }}=2,23 ; \mathrm{P}_{\text {semillas }}=0,52$, Tabla 2 ).

El Índice de Autoincompatibilidad (IAI) basado en el número de semillas por fruto fue de $0,13 \pm 0,07$ (media \pm 1EE) y no difirió significativamente de 0 (test de Wald: $\mathrm{t}=1,92 ; \mathrm{gl}=10 ; \mathrm{p}=0,08)$, mientras que la eficiencia reproductiva (ER) fue $1,82 \pm 0,07$ (media $\pm 1 \mathrm{EE}$ ) y no difirió de 1,0 (test de Wald: $\mathrm{t}=1,45 ; \mathrm{gl}=9 ; \mathrm{p}=0,18$ ). El índice de autofertilidad (IAF) fue $0,18 \pm 0,13$ (media $\pm 1 \mathrm{EE}$ ) $\mathrm{y}$ no difirió significativamente de 0 (test de Wald: $\mathrm{t}=1,44$; $\mathrm{gl}=10 ; \mathrm{p}=0,18)$.

Tabla 1. Ensayos de receptividad del estigma según edad floral. La fase floral se señala en el texto. Diferentes letras indican diferencias significativas entre tratamientos evaluado con test de Tukey. / Essays of stigma receptivity according to floral age. Different letters indicate significant differences between treatments evaluated with Tukey's test.

\begin{tabular}{|c|c|c|c|c|c|c|}
\hline TRATAMIENTO & FASE FLORAL & $\begin{array}{l}\mathrm{N}^{\mathrm{o}} \text { PLANTAS } \\
\text { UTILIZADAS }\end{array}$ & $\begin{array}{l}\mathrm{N}^{\mathrm{o}} \text { FLORES } \\
\text { UTILIZADAS }\end{array}$ & $\begin{array}{l}\mathrm{N}^{\mathrm{o}} \text { FRUTOS } \\
\text { FORMADOS }\end{array}$ & $\begin{array}{c}\% \text { DE } \\
\text { FRUCTIFICACIÓN }\end{array}$ & $\begin{array}{c}\mathrm{N}^{\mathrm{o}} \text { SEMILLAS POR FRUTO } \\
(\text { MEDIA } \pm 1 \mathrm{EE})\end{array}$ \\
\hline Día 0 & $\mathrm{~F} 1$ & 4 & 4 & 4 & 1,00 & $50,00 \pm 15,09 \mathrm{a}$ \\
\hline Día 1 & $\mathrm{~F} 1$ & 4 & 6 & 4 & 0,67 & $44,75 \pm 11,89 \mathrm{a}$ \\
\hline Día 3 & $\mathrm{~F} 2$ & 4 & 7 & 7 & 1,00 & $67,63 \pm 15,93 \mathrm{a}$ \\
\hline Día 6 & $\mathrm{~F} 2$ & 4 & 5 & 4 & 0,80 & $42,50 \pm 12,78 \mathrm{a}$ \\
\hline Día 7 & $\mathrm{~F} 2$ & 4 & 5 & 5 & 1,00 & $43,00 \pm 11,92 \mathrm{a}$ \\
\hline Día 8 & F3 & 4 & 4 & 2 & 0,50 & $3,00 \pm 2,12 b$ \\
\hline
\end{tabular}


TABLA 2. Ensayos de eficiencia reproductiva según tipo de polinización. Diferentes letras indican diferencias significativas entre tratamientos con test de Tukey. / Reproductive efficiency tests by type of pollination. Different letters indicate significant differences between treatments with Tukey's test.

\begin{tabular}{|c|c|c|c|c|c|}
\hline TRATAMIEnto & $\begin{array}{l}\mathrm{N}^{\mathrm{o}} \text { PLANTAS } \\
\text { UTILIZADAS }\end{array}$ & $\begin{array}{l}\mathrm{N}^{\mathrm{o}} \text { FLORES } \\
\text { UTILIZADAS }\end{array}$ & $\begin{array}{l}\mathrm{N}^{\mathrm{o}} \text { FRUTOS } \\
\text { FORMADOS }\end{array}$ & $\begin{array}{l}\text { FRUCTIFICACIÓN } \\
(\text { MEDIA } \pm 1 \text { EE) }\end{array}$ & $\begin{array}{c}\mathrm{N}^{\mathrm{o}} \text { SEMILLAS POR FRUTO } \\
(\text { MEDIA } \pm 1 \mathrm{EE})\end{array}$ \\
\hline AGA & 11 & 32 & 1 & $0,05 \pm 0,05 \mathrm{a}$ & $0,14 \pm 0,27 \mathrm{a}$ \\
\hline AUTO & 11 & 33 & 3 & $0,09 \pm 0,07 \mathrm{a}$ & $0,67 \pm 1,18 \mathrm{a}$ \\
\hline APM & 11 & 32 & 14 & $0,44 \pm 0,08 \mathrm{~b}$ & $2,62 \pm 3,78 \mathrm{a}$ \\
\hline PCM & 11 & 32 & 24 & $0,74 \pm 0,08 \mathrm{c}$ & $37,26 \pm 17,57 b$ \\
\hline PN & 11 & 73 & 72 & $0,98 \pm 0,01 \mathrm{c}$ & $47,61 \pm 11,45 b$ \\
\hline
\end{tabular}

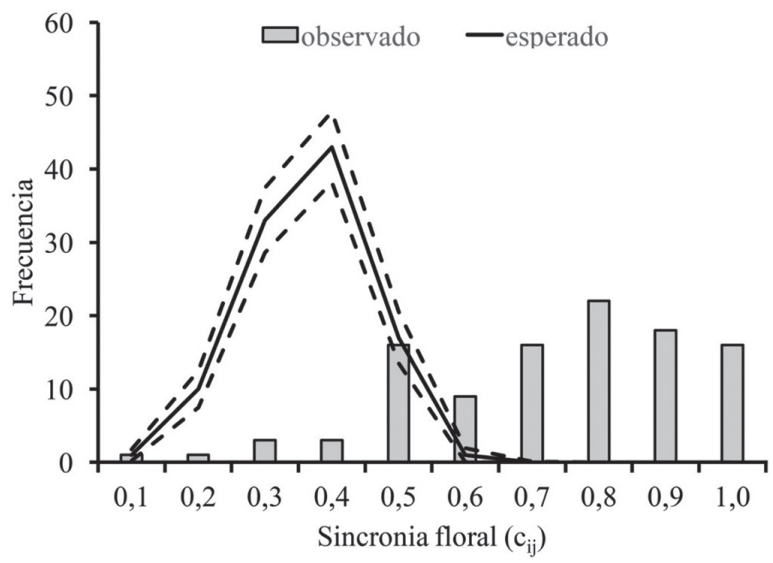

Figura 2. Distribución observada (en barras) y esperada (en línea negra) de sincronía floral $\left(\mathrm{c}_{\mathrm{ij}}\right)$ entre plantas. Las líneas segmentadas corresponden a los intervalos de confianza al $95 \%$ para la sincronía floral esperada obtenida de 100 permutaciones. / Observed (bars) and expected distribution (black line) of floral synchrony between plants $\left(\mathrm{c}_{\mathrm{ij}}\right)$. Segmented lines correspond to the $95 \%$ confidence intervals for the expected distribution obtained from 100 permutations.

\section{Distancia A LA PLANTA DONANTE}

Las plantas polinizadas manualmente con polen proveniente de plantas ubicadas a diferentes distancias presentaron diferencias significativas en la producción de semillas por fruto (AMR: $F(3,30)=23,53 ; p<0,001)$. Estas diferencias se debieron a la disminución del número de semillas cuando el polen era de la misma planta $(0 \mathrm{~m})$ respecto a cualquier otra distancia a la planta donante (Tabla 3). Sin embargo, los tratamientos de polinización cruzada manual con polen proveniente de plantas a 5,30 y $1.000 \mathrm{~m}$ de la planta receptora no presentaron diferencias significativas entre ellas (test de Tukey, $\mathrm{p}>0,13$ en todos los casos). Al comparar la fracción de flores que fructificaron, se obtuvieron similares resultados $(\mathrm{AMR}$ : $\mathrm{F}(3,30)=3,83 ; \mathrm{p}=$ 0,019), sin embargo solo se encontró una mayor fracción de frutos formados a una distancia de $30 \mathrm{~m}$ respecto a $0 \mathrm{~m}$ (test de Tukey, $Q=4,60 ; p=0,014)$.
FRECUENCIA DE VISITANTES FLORALES

En tres temporadas se detectaron 95 especies de insectos visitando las flores, pero sólo una pequeña fracción $(23,2 \%$ del total de especies) presentó una alta frecuencia de visitas durante 3 años ( $>90 \%$ de las visitas, Tabla 4$)$. De las 95 especies, los himenópteros fueron los más representados con 38 especies $(40,0 \%)$, seguidos por dípteros con 28 especies (29,5\%), luego coleópteros con 17 especies $(17,9 \%)$ y al final lepidópteros con 12 especies (12,6\%). En relación a la frecuencia de visitas y considerando los tres años, los himenópteros realizaron el $36,6 \%$ del total de visitas a las flores, los coleópteros el 56,6\%, los dípteros el 3,0\% y los lepidópteros el 3,8\% del total de visitas observadas a las flores. En relación a los visitantes nocturnos, sólo dos especies de polillas $(2,1 \%$ del total de especies) realizaron el $11,7 \%$ del total de visitas, mientras que todo el resto de las visitas $(88,3 \%)$ fueron realizadas por visitantes diurnos.

La especie que con mayor frecuencia visitó las flores fue la abeja Apis mellifera, que alcanzó más del 45\% del total de visitas a las flores en las dos últimas temporadas (ver Tabla 4). Dentro de los insectos nativos se destacan los coleópteros Amecocerus sp., Cylindrophora maulica y Mordella proxima, los que fueron consumidores de polen en las flores y presentaron un bajo nivel de movimiento entre plantas. Dentro de los dípteros se observaron tres tipos de consumidores: 1) especies con aparato bucal largo no retráctil, que sólo ingresan el aparato bucal a las flores y consumen néctar, como Trichophthalma subaurata y Scaptia viridiventris, 2) especies con aparato bucal retráctil y que actúan como consumidoras de polen y néctar como Chaetocnephalia sp., Archytas scutellatus y Villa sp., las que deben ingresar parte de la cabeza a las flores, 3) especies pequeñas ( $<5 \mathrm{~mm}$ de largo), las que deben ingresar el cuerpo completo a la flor para lamer néctar, como Megalybus crassus y Phthiria austrandina. Entre los himenopteros, se observaron tres tipos de consumidores: 1) abejas nativas de tamaño mediano $(\sim 10 \mathrm{~mm}$ de largo, como Apis mellifera) que recolectaron néctar y polen ingresando solo la cabeza a las flores, como Colletes nigritulus y Lonchopria zonalis, 2) Avispas de tamaño 
grande $(>10 \mathrm{~mm})$ consumidoras de néctar, como Sphex latreillei, las que sólo ingresaron una pequeña porción de la cabeza a las flores, y 3) abejas pequeñas consumidoras de néctar y polen, las que deben ingresar su cuerpo completo a las flores, como Lasioglossum herbstiellus, Calliopsis trifasciata y Caenohalictus aplacodes. Por último, entre los lepidópteros destacan dos especies, una diurna (Auca delessei) y otra nocturna (Copitarsia decolora). La primera realiza cortas visitas ( $<1 \mathrm{~min})$ a pocas flores de una misma planta, realizando vuelos entre plantas distantes. La segunda permanece durante largos periodos de tiempo ( $>1 \mathrm{~min}$ ) en la inflorescencia, libando el néctar de varias flores con la espiritrompa, para luego ir a otra planta. En ambos casos la espiritrompa es la principal estructura que entra en contacto con las partes reproductivas de la flor al acceder al nectario.

Tabla 3. Ensayos de efíciencia reproductiva según la distancia a la planta donante de polen. Diferentes letras indican diferencias significativas entre tratamientos con test de Tukey. / Reproductive efficiency tests according to the pollen donor plant distance. Different letters indicate significant differences between treatments with Tukey's test.

\begin{tabular}{|c|c|c|c|c|c|}
\hline TRATAMIENTO & $\begin{array}{l}\mathrm{N}^{\mathrm{o}} \text { PLANTAS } \\
\text { UTILIZADAS }\end{array}$ & $\begin{array}{l}\mathrm{N}^{\mathrm{o}} \text { FLORES } \\
\text { UTILIZADAS }\end{array}$ & $\begin{array}{l}\mathrm{N}^{\mathrm{o}} \text { FRUTOS } \\
\text { FORMADOS }\end{array}$ & $\begin{array}{l}\text { FRUCTIFICACIÓN } \\
(\text { MEDIA } \pm 1 \text { EE) }\end{array}$ & $\begin{array}{l}\mathrm{N}^{\mathrm{o}} \text { SEMILLAS POR FRUTO } \\
(\text { MEDIA } \pm 1 \mathrm{EE})\end{array}$ \\
\hline $0 \mathrm{~m}$ & 11 & 32 & 14 & $0,44 \pm 0,08 \mathrm{a}$ & $2,62 \pm 1,93 \mathrm{a}$ \\
\hline $5 \mathrm{~m}$ & 11 & 32 & 24 & $0,74 \pm 0,08 \mathrm{ab}$ & $37,26 \pm 8,96 b$ \\
\hline $30 \mathrm{~m}$ & 11 & 32 & 27 & $0,85 \pm 0,05 \mathrm{~b}$ & $54,76 \pm 11,73 b$ \\
\hline $1.000 \mathrm{~m}$ & 11 & 33 & 22 & $0,67 \pm 0,11 \mathrm{ab}$ & $36,30 \pm 11,92 b$ \\
\hline
\end{tabular}

TABLA 4. Frecuencia de visitantes florales de E. pulverulenta. En negrita se destacan las especies más frecuentes durante tres años (Los asteriscos $\left(^{*}\right)$ son datos no registrados). / Frequency of floral visitors of E. pulverulenta. In bold the most common species during three years stand out. (Asterisk $(*)$ are unregistered data).

\begin{tabular}{|c|c|c|c|c|c|}
\hline \multirow{2}{*}{ ORDEN } & \multirow[b]{2}{*}{ FAMILIA } & \multirow[b]{2}{*}{ ESPECIE } & \multicolumn{3}{|c|}{ FrecuenCia RELATIVA (\%) } \\
\hline & & & 2013 & 2014 & 2015 \\
\hline \multirow[t]{7}{*}{ Coleoptera } & Buprestidae & Cylindrophora maulica & $\mathbf{0 , 2 0}$ & 0,96 & $\mathbf{0 , 1 5}$ \\
\hline & Cleridae & Epiclines gayi & 0,10 & 0,21 & 0,97 \\
\hline & Dermestidae & Trogoderma vicinum & 1,10 & 0,00 & 2,67 \\
\hline & Melyridae & Amecocerus sp. & 68,90 & 4,17 & 18,86 \\
\hline & Mordellidae & Mordella proxima & $\mathbf{0 , 5 0}$ & $\mathbf{0 , 8 8}$ & 2,75 \\
\hline & Scarabeidae & Arctodium vulpinum & 0,03 & 0,19 & 0,45 \\
\hline & otros & +11 especies & 1,30 & 3,46 & 1,43 \\
\hline \multirow[t]{8}{*}{ Diptera } & Acroceridae & Megalybus crassus & 0,30 & 0,05 & 0,00 \\
\hline & Bombyliidae & Phthiria austrandina & 0,80 & 0,35 & 0,00 \\
\hline & Bombyliidae & Villa $s p$ & 0,40 & 0,08 & 0,11 \\
\hline & Calliphoridae & Sarconesia dichroa & 0,10 & 0,00 & 0,36 \\
\hline & Nemestrinidae & Trichophthalma subaurata & $\mathbf{0 , 5 0}$ & 0,77 & $\mathbf{0 , 0 7}$ \\
\hline & Tabanidae & Scaptia sp. & 0,10 & 0,35 & 0,06 \\
\hline & Tachinidae & Chaetocnephalia sp. & 0,10 & 0,08 & 0,00 \\
\hline & Otros & +21 especies & 1,30 & 0,90 & 0,12 \\
\hline \multirow[t]{8}{*}{ Hymenoptera } & Andrenidae & Calliopsis trifasciata & 0,20 & 0,08 & 0,06 \\
\hline & Apidae & Apis mellifera & 11,70 & 71,89 & 56,42 \\
\hline & Colletidae & Colletes nigritulus & 1,20 & $\mathbf{0 , 8 5}$ & $\mathbf{0 , 0 6}$ \\
\hline & Colletidae & Lonchopria zonalis & 0,60 & 0,98 & $\mathbf{0 , 0 7}$ \\
\hline & Halictidae & Caenohalictus aplacoides & 0,20 & 0,82 & 0,04 \\
\hline & Halictidae & Lasioglossum herbstiellus & 2,00 & $\mathbf{0 , 8 2}$ & 0,28 \\
\hline & Sphecidae & Sphex latrellei & 1,80 & 6,59 & 1,46 \\
\hline & otros & +31 especies & 4,90 & 2,59 & 0,52 \\
\hline \multirow[t]{4}{*}{ Lepidoptera } & Noctuidae & Copitarsia decolora & * & $*$ & 10,29 \\
\hline & Nymphalidae & Auca delessei & 1,60 & 2,84 & 1,08 \\
\hline & otros & +10 especies & 0,07 & 0,09 & 1,72 \\
\hline & Total & 95 especies & 100,0 & 100,0 & 100,0 \\
\hline
\end{tabular}




\section{DISCUSIÓN}

Sistema REPRODUCTIVO

De acuerdo al valor del índice de autoincompatibilidad obtenido para Escallonia pulverulenta, esta especie debe ser considerada autoincompatible (Arroyo \& Squeo 1990). La restricción que presentan los individuos para autofecundarse se expresa en el bajo número de semillas por fruto obtenido en la prueba de autopolinización manual $(\mathrm{IAI}=0,13)$ y la baja producción de semillas obtenida en la prueba de autopolinización automática (IAF=0,18). La autoincompatibilidad observada en E. pulverulenta es coherente con la observada en Escallonia myrtoidea (Valdivia \& Niemeyer 2006) y con la tendencia general de que las formas de vida arbustivas y arbóreas son autoincompatibles (Barrett 2002). En el caso de plantas leñosas chilenas, un $75 \%$ de las especies arbustivas en la flora alpina patagónica chilena son autoincompatibles (Arroyo \& Squeo 1990), al igual que el $80 \%$ de los arbustos estudiados en el bosque esclerófilo montano de los Andes de Chile central (Arroyo \& Uslar 1993). Su condición de autoincompatibilidad implica que requieren la intervención de un agente polinizador biótico y que estos polinizadores deben visitar diferentes individuos, no sólo flores de una misma planta, para realizar polinización cruzada efectiva y contribuir con la reproducción de la planta.

Por otro lado, el similar número de semillas obtenidas por polinización natural respecto de la polinización cruzada manual $(E R=1,83)$ sugiere que la producción de semillas en E. pulverulenta no está limitada por la actividad de los polinizadores (Bierzychudek 1981). Aunque el movimiento de forrajeo realizado por dos de los visitantes más frecuentes, Apis mellifera y Amecocerus sp. (Tabla 4) fue visitar varias flores dentro de un mismo individuo, lo que puede aumentar la incidencia de polinización geitonogámica, es posible que la actividad de las otras especies que presentan mayor movimiento entre plantas, como las mariposas, polillas y abejas nativas, eleven los niveles de xenogamia en las flores. Otra explicación no excluyente es que la polinización cruzada manual haya resultado con similar número de semillas que la polinización natural debido a una aplicación excesiva de polen o por repartirla de forma heterogénea en la superficie del estigma, interfiriendo en el desarrollo de los tubos polínicos (i.e. interferencia estigmática, Young \& Young 1992).

En relación a los efectos temporales y espaciales en el éxito reproductivo de E. pulverulenta, se determinó que el número de días de la flor en antesis (durante los primeros 7 días en fase F1 y F2) y la distancia de origen del polen entre plantas vecinas (de $5 \mathrm{~m}$ a $1.000 \mathrm{~m}$ ), no modifican el éxito reproductivo de E. pulverulenta. De esta forma, los polinizadores pueden visitar las flores varios días durante los 5 días de la fase funcional para la polinización (fase F2: flor homógama) y su contribución a la producción de semillas podría acumularse con la mayor deposición de polen xenógamo en los estigmas. A su vez, aun cuando se ha observado que mariposas y abejas difieren en las distancias de vuelo post visita y por tanto en el flujo de genes dentro y entre poblaciones (Herrera 1987), las abejas que recorren distancias cortas entre plantas $(<5 \mathrm{~m})$, o las mariposas que recorren largas distancias entre plantas $(>15$ $\mathrm{m})$, podrían ser igualmente efectivas como polinizadores de E. pulverulenta, siempre y cuando el polen recolectado sea depositado en una planta diferente, ya sea vecina (dentro de $5 \mathrm{~m}$ ) o alejada (a $1 \mathrm{~km})$. Esto también estaría sugiriendo que no hay una estructuración genética espacial entre los individuos (Glaettli et al. 2006), al menos en el rango de distancia considerado (de $5 \mathrm{~m}$ a $1 \mathrm{~km}$ ).

FLORACIÓN Y BIOLOGÍA FLORAL ASOCIADOS AL SISTEMA REPRODUCTIVO

E. pulverulenta se ajusta a un patrón de floración en masa (De Jong et al. 1992), ya que produce un gran número de flores pequeñas en inflorescencias que se abren de forma sincrónica entre individuos, lo que se ha señalado como una estrategia que permite atraer a un gran número de polinizadores pero que implican altos niveles de geitonogamia (Augspurger 1983, De Jong et al. 1992). Por otro lado, dentro de una inflorescencia las flores se abren asincrónicamente, desde la base hacia el ápice y duran varios días abiertas ( $\sim 5$ días), por lo que en una misma inflorescencia se pueden observar flores en diferentes etapas de desarrollo, lo que promovería la polinización cruzada (Primack 1985, De Jong et al. 1992, Harder \& Barrett 1995). Al respecto, Thomson et al. (2000) proponen que plantas especializadas en la polinización por abejas, las cuales generalmente tienen altas tasas de recolección de polen pero bajas tasas de deposición en los estigmas, debiesen presentar algunos atributos florales que maximicen la polinización cruzada, tales como: 1) presentación gradual del polen en las anteras, en bajas cantidades y lentamente, 2) un bajo número de anteras, 3 ) estigmas y anteras insertas (i.e. no sobresalen de la flor), 4) recursos florales expuestos al polinizador, 5) presencia de zonas de aterrizaje, y 6) néctar concentrado y en pequeñas cantidades. En el caso de E. pulverulenta se observan todos estos atributos: 1) alta longevidad floral (cinco días de duración), 2) número de anteras igual al número de pétalos y sépalos, 3) gineceo y androceo de longitud similar y al mismo nivel que la corola, 4) nectario visible de color amarillo que contrasta con la corola de pétalos blancos, 5) la inflorescencia actúa como zona de aterrizaje (Fig. 1a), y 6) néctar ofertado en bajas dosis y de apariencia viscosa (ver también Díaz-Forestier et al. 2016 para detalles del nectario), lo que sugiere algún nivel de especialización a la polinización por abejas, además de ser los visitantes florales que más frecuentemente se observaron. Por otro lado, Valdivia \& Niemeyer (2006) propusieron que los 
caracteres florales de E. mytoidea, como flores pequeñas agrupadas en inflorescencias, la emisión de aromas dulces, la producción de néctar como recompensa y la apertura de las flores durante el día y la noche, indicarían una polinización mediada por mariposas y polillas. Aunque varios de esos caracteres se parecen a los de E. pulverulenta, la importancia relativa de los Lepidópteros en términos de su frecuencia de visitas fue menor que la de cualquier otro orden de insecto (al igual que en E. myrtoidea), sugiriendo un rol menor en el éxito reproductivo de E. pulverulenta, lo que no excluye la posibilidad que las mariposas hayan sido en el pasado los principales polinizadores de estas plantas (Valdivia \& Niemeyer 2006). Junto con esto, la apertura nocturna de las flores de E. pulverulenta puede seguir siendo en la actualidad un atributo adaptativo si Copitarsia decolora (Noctuidae), el principal visitante nocturno, es además un polinizador eficiente y si el efecto conjunto de la polinización nocturna más la diurna resulta en una mayor adecuación biológica para la planta respecto a presentar solo polinizadores diurnos. De todas formas, se esperaría que la contribución de los visitantes diurnos debiese ser mayor que los nocturnos, por su mayor diversidad y frecuencia de visitas.

\section{VisITANTES FLORALES}

En relación a los visitantes florales, debido a los cambios interanuales registrados en la frecuencia de insectos que visitaron las flores de E. pulverulenta (Tabla 4), estos serían temporalmente impredecibles, lo que favorecería la mantención de un ensamble generalista de polinizadores (Gómez 2002). Bajo este escenario, las poblaciones de E. pulverulenta podrían asegurar algún nivel mínimo de reproducción si los insectos que dejan de visitar las flores entre años son reemplazados por otros con efectividades equivalentes. A su vez, esta variabilidad interanual de potenciales polinizadores puede deberse a cambios anuales en las condiciones climáticas, lo que afectaría de manera diferencial a las poblaciones de insectos, favoreciendo a algunas especies por sobre otras. Otra posible fuente de variación interanual podría deberse a la actividad de la abeja eusocial Apis mellifera, la que supera ampliamente en número de individuos a todas las especies de abejas nativas. Esta especie puede afectar las visitas de otras especies de polinizadores nativos por acaparamiento y reducción de los recursos florales, ya que al llegar varios individuos a una misma planta, disminuirían los recursos florales disponibles para otras especies, además de aumentar los niveles de polinización geitonógama por concentrar sus visitas en flores de una misma planta (Valido et al. 2014), traduciéndose en una disminución en la producción de semillas en especies autoincompatibles, como E. pulverulenta. Al respecto, algunas observaciones en zonas dentro de la reserva donde se observa baja actividad de la abeja doméstica, coinciden con un incremento de las visitas por otras especies de abejas nativas (obs. pers.). Junto con esto, los dos últimos años que incrementó la frecuencia de visita de $A$. mellifera a las flores de $E$. pulverulenta, disminuyó la representación de otras especies de abejas como Calliopsis trifasciata, Colletes nigritulus, Lasioglossum herbstiellus y la mosca Chaetocnephalia sp. (Tabla 4). Si esta disminución se debió al incremento de la abeja doméstica y/o factores climáticos, se desconoce y requiere posteriores estudios por los potenciales impactos negativos que podría tener $A$. mellifera en los polinizadores y la flora nativa, como se ha documentado en sistemas insulares (Valido et al. 2014).

La alta diversidad taxonómica de insectos que visitan las flores de E. pulverulenta también se traduce en una alta diversidad funcional, ya que las especies que visitan las flores difieren en sus tamaños corporales, conductas de forrajeo (e.g. constancia floral y hábitos de limpieza), tiempo de permanencia en las flores, número de flores visitadas por planta y la frecuencia de vuelo entre plantas, todas las cuales afectan las tasas de transferencia de polen xenógamo y los niveles de descuento de polen (Herrera 1987, Zimmerman 1988, Barret 2003). Se desconoce qué tan equivalentes o diferentes son las eficiencias de las especies de insectos, pero se esperaría que fuesen similares entre especies pertenecientes a un mismo grupo funcional (Gómez \& Zamora 1999). Finalmente, la diversidad taxonómica y funcional de visitantes florales observadas en E. pulverulenta también fue observada en E. myrtoidea (Valdivia \& Niemeyer 2006), por lo que posiblemente esta sea una característica compartida con otras especies del género.

\section{AGRADECIMIENTOS}

Este estudio fue financiado por proyecto FONDECYT de iniciación No11130429 otorgado a Antonio RiveraHutinel. Se agradece a CONAF y los Guardaparques de Río Clarillo por las facilidades para realizar el estudio y a Jaime Solervicens, Christian González, Patricia Estrada, Francisco Urra y Marcos Beèche por la ayuda en la identificación de los insectos.

\section{REFERENCIAS}

Arroyo, M.T.K., Squeo, F.A. 1990. Relationship between plant breeding systems and pollination. In: Kawano, S. (ed.). Biological Approaches and Evolutionary Trends in Plants. pp. 205-227. Academic Press, London, UK.

Arroyo, M.T.K., Uslar, P. 1993. Breeding systems in a temperate mediterranean-type climate montane sclerophyllous forest in central Chile. Botanical Journal of the Linnean Society 111: 83-102.

Augspurger, C.K. 1983. Phenology, flowering synchronity and fruit set of six neotropical shrubs. Biotropica 15: 257-267. 
BARRETT, S.C.H. 2002. The evolution of plant sexual diversity. Nature Reviews Genetics 3: 274-284.

BARRETT, S.C.H. 2003. Mating strategies in flowering plants: the outcrossing-selfing paradigm and beyond. Philosophical Transactions of the Royal Society of London. Serie B 358: 991-1004.

BERTIN, R. 1993. Incidence of monoecy and dichogamy in relation to self-fertilization in angiosperms. American Journal of Botany 80: 557-560.

BierzychudeK, P. 1981. Pollinator limitation of plant reproductive effort. American Naturalist 117: 838-840.

Byers, D. 1998. Effect of cross proximity on progeny fitness in a rare and a common species of Eupatorium (Asteraceae). American Journal of Botany 85(5): 644-653.

Charlesworth, D. 2002. Self-incompatibility: how to stay incompatible. Current Biology 12: R424-R426.

De Jong, T.J., Klinkhamer, P.G.L., Van Staalduinen, M.J. 1992. The Consequences of Pollination Biology for Selection of Mass or Extended Blooming. Functional Ecology 6(5): 606-615.

Díaz-Forestier, J., Gómez, M., Celis-Diez, J.L., Montenegro, G. 2016. Nectary structure in four melliferous plant species native to Chile. Flora 221: 100-106.

Eckert, C.G., Barrett, S.C.H. 1994. Post-pollination mechanisms and the maintenance of outcrossing in self-compatible, tristylous, Decodon verticillatus (Lythraceae). Heredity 72: 396-411.

Fox, J. 2002. An R and S-Plus Companion to Applied Regression. SAGE Publications, London. 311 pp.

Glaettli, M., Pescatore, L., \& Goudet, J. 2006. Proximitydependent pollen performance in Silene vulgaris. Annals of Botany 98: 431-437.

Glover, B.J. 2014. Understanding Flowers and Flowering: An Integrated Approach. Oxford University Press, Second Edition, New York. 227 pp.

Gómez, J.M. 2002. Generalización en las interacciones entre plantas y polinizadores. Revista Chilena de Historia Natural 75: 105-116.

Gómez, J.M., ZAMORA, R. 1999. Generalization vs. specialization in the pollination system of Hormathophylla spinosa (Cruciferae). Ecology 80(3): 796-805.

HAMmer, Ø., Harper, D.A.T., RYAN, P.D. 2001. PAST: Paleontological statistics software package for education and data analysis. Palaeontologia Electronica 4(1): 9.

Harder, L.D., Barrett, S.C.H. 1995. Mating cost of large floral displays in hermaphrodite plants. Nature 373: 512-515.

Hardner, C.M., Potts, B.M., Gore, P.L. 1998. The relationship between cross success and spatial proximity of Eucalyptus globosus ssp. globulus parents. Evolution 52(2): 614-618.

Herrera, C.M. 1987. Components of pollinator "quality": comparative analysis of a diverse insect assemblage. Oikos 50: 79-90.

Herrera, C.M. 2005. Plant generalization on pollinators: species property or local phenomenon? American Journal of Botany 92(1): 13-20.

Hoffmann, A. 2012. Flora silvestre de Chile: zona central. Ediciones Fundación Claudio Gay, Quinta Edición, Santiago. 254 pp.

Hurlbert, S.H. 1984. Pseudoreplication and the design of ecological field experiments. Ecological Monographs
54(2): 187-211

Kausel, E.M.L. 1953. Revisión del género Escallonia en Chile. Darwiniana 10(2): 169-255.

Kearns, C.A., Inouye, D.W. 1993. Techniques for Pollination Biologists. University Press of Colorado. Colorado, USA. $583 \mathrm{pp}$

Lloyd, C.G., Schoen, D.J. 1992. Self and cross-fertilizations on plants. I. Functional dimensions. International Journal of Plant Science 153(3): 358-369.

McClure, B., Franklin-Tong, V. 2006. Gametophytic selfincompatibility: understanding the cellular mechanisms involved in 'self' pollen tube inhibition. Planta 224: 233245.

Ministerio del Medio Ambiente. 2016. Inventario nacional de especies de Chile. URL: http://especies.mma.gob.cl/. Viewed: Octubre 10, 2016.

Montenegro, G., Peña, R.C., Pizarro, R. 2010. Multivariate analysis of pollen frequency of the native species Escallonia pulverulenta (Saxifragaceae) in Chilean honeys. Revista Brasilera de Botánica 33(4): 615-630.

Primack, R.B. 1980.Variation in the phenology of natural populations of montane shrubs in New Zealand. Journal of Ecology 68(3): 849-862

Primack, R.B. 1985. Longevity of Individual Flowers. Annual Review of Ecology and Systematics 16: 15-37.

R DEVELOPMENT CORE TEAM. 2009. R: A language and environment for statistical computing. R Foundation for Statistical Computing, Vienna, Austria. URL: http:// www.R-project.org.

Ruiz-Zapata, T., Arroyo, M.T.K. 1978. Plant reproductive ecology of a secondary deciduous forest in Venezuela. Biotropica 10: 221-230.

Sargent, R.D., Оtтo, S.P. 2004. A phylogenetic analysis of pollination mode and the evolution of dichogamy in angiosperms. Evolutionary Ecology Research 6: 11831199.

Schneider, C.A., Rasband, W.S., Eliceiri, K.W. 2012. NIH Image to ImageJ: 25 years of image analysis. Nature Methods 9: 671-675.

Sleumer, H.O. 1968. Die Gattung Escallonia (Saxifragaceae). Verhandelingen der Koninklijke Nederlandse Akademie van Wetenschappen, Afd. Natuurkunde 58: 1-146.

Schmitt, J., Gamble, S. 1990. The effect of distance from the parental site on offspring performance and inbreeding depression in Impatiens capensis: A test of a local adaptation hypothesis. Evolution 44(8): 2022-2030.

Takebayashi, N., Wolf, D., Delph, L. 2006. Effect of variation in herkogamy on outcrossing within a population of Gilia achilleifolia. Heredity 96:159-165.

Teillier, S., Aldunate, G., Riedemann, P., Niemeyer, H. 2005. Flora de la Reserva Nacional Río Clarillo: Guía de Identificación de Especies. Impresos Socías, Santiago. 367 pp.

The Plant List. 2013. Version 1.1. Published on the Internet; http://www.theplantlist.org/ (accessed 1st January).

Thomson, J.D., Wilson, P., Valenzuela, M., Malzone, M. 2000. Pollen presentation and pollination syndromes, with special reference to Penstemon. Plant Species Biology 15: 11-29.

VAldivia, C.E., NiemEYer, H.M. 2006. Do floral syndromes predict specialisation in plant pollination systems? Assessment of 
diurnal and nocturnal pollination of Escallonia myrtoidea. New Zealand Journal of Botany 44: 135-141.

Valido, A., Rodríguez-Rodríguez, M.C., Jordano, P. 2014. Impacto de la introducción de la abeja doméstica (Apis mellifera, Apidae) en el Parque Nacional del Teide (Tenerife, Islas Canarias). Ecosistemas 23(3): 58-66.

YounG, H.J., Young, T.P. 1992. Alternative Outcomes of Natural and Experimental High Pollen Loads. Ecology 73(2): 639-647.

Yumoto, T. 1987. Pollination in the canopy: two corollaries deduced from the energetics of pollination. In: Kawano,
S., Connell, J.H., Hidaka, T. (eds.), Evolution and coadaptation in biotic communities. pp. 165-182. Tokio Press, Tokio, Japan.

ZAPATA, F. 2013. A multilocus phylogenetic analysis of Escallonia (Escalloniaceae): diversification in montane South America. American Journal of Botany 100(3): 526-545.

Zimmerman, M. 1988. Nectar production, flowering phenology, and strategies for pollination. In: Lovett Doust, J., Lovett Doust, L. (eds.). Plant Reproductive Ecology. Patterns and Strategies. pp. 157-178. Oxford University Press, New York, USA.

Recibido: 14.11 .2016

Aceptado: 21.04.2017 